\title{
Interdisziplinäres Management bei fortgeschrittener Leberzirrhose
}

\author{
Stefan Zeuzem ${ }^{a}$ Wolf O. Bechstein ${ }^{b}$ \\ ${ }^{\text {a }}$ Medizinische Klinik 1, \\ ${ }^{\mathrm{b}}$ Klinik für Allgemein- und Viszeralchirurgie, Klinikum der Johann Wolfgang Goethe-Universität, Frankfurt/M., Deutschland
}

Patienten mit fortgeschrittener Leberzirrhose können eine Vielzahl von komplexen medizinischen Problemen entwickeln, die eine Betreuung durch ein interdisziplinäres Team erforderlich machen. Hauptbehandlungspartner dieses Teams sind neben der Gastroenterologie und Hepatologie die Viszeral- und Transplantationschirurgie sowie die diagnostische und interventionelle Radiologie. Die beste Therapie für viele Patienten mit fortgeschrittener Leberzirrhose ist die Lebertransplantation. Hierdurch kann schwer kranken Patienten, die eine extrem hohe 1-Jahres-Mortalität aufweisen, ein Langzeitüberleben ermöglicht werden. Leider ist die Zahl an Spenderorganen in unserem Land gemessen an der Anzahl der Patienten auf einer Lebertransplantationswarteliste zu gering. Die Vorfälle in Göttingen, bei denen persönliches Fehlverhalten von einzelnen Ärzten zur unrechtmäßigen Vergabe von Organen führte, hat leider dazu beitragen, dass die Lebertransplantation in Deutschland in die Negativschlagzeilen geraten ist. Obwohl die Organspende, -vermittlung und -transplantation in Deutschland durch das Transplantationsgesetz organisatorisch streng voneinander getrennt sind, ist es zum aktuellen Transplantationsskandal gekommen. Hätte der Skandal verhindert werden können? Nach unserer Auffassung ja. Eine interdisziplinäre Behandlung mit transparenter Kommunikation der erhobenen Befunde an alle Behandlungspartner, die zuweisenden Ärzte und vor allem auch den Patienten einschließend, stellt eine hohe Barriere dar, vor der es nur sehr schwer zu solchen Ereignissen wie in Göttingen kommen kann. Die Interdisziplinarität sollte hierbei nicht nur informellen Charakter aufweisen, sondern, wie in großen Transplantationszentren bereits üblich, in Strukturen gefasst werden - mit der Bildung von «Leberzentren», gemeinsamen Visiten und wöchentlichen interdisziplinären Konferenzen, in denen relevante Therapieentscheidungen gemeinsam getroffen werden und das Ergebnis schriftlich fixiert und auch kommuniziert wird. Ein weiterer Schritt zur Steigerung der Trans- parenz sind regelmäßige Morbiditäts- und Mortalitätskonferenzen, im angloamerikanischen Raum schon lange Standard, bei denen offen über Komplikationen diskutiert wird. Der nächste konsequente Schritt ist die unabhängige Prüfung von Lebertransplantationszentren in Form von Audits und eine Zertifizierung, wie sie in der Onkologie schon seit etlichen Jahren Standard ist.

In diesem Themenheft stellen Experten aus den Lebertransplantationszentren Essen, München und Frankfurt/M. aktuelle Aspekte der interdisziplinären Behandlung von Patienten mit fortgeschrittener Leberzirrhose in Form von Übersichtsarbeiten dar. Die häufigsten Komplikationen bei Patienten mit fortgeschrittener Zirrhose sind das Auftreten von Aszites, einer Pfortaderthrombose und einer hepatischen Enzephalopathie. Gerbes und Gülberg [1], München, zeigen in ihrem Beitrag die aktuellen Therapieempfehlungen bei diesen Komplikationen auf. Wir wissen heute sehr gut, dass die effektive Behandlung einer chronischen Hepatitis-B- und Hepatitis-C-Virusinfektion vor der Transplantation maßgeblich für den Langzeitverlauf dieser Patienten mit verantwortlich sind. Farnik und Sarrazin [2], Frankfurt/M., fassen im zweiten Beitrag die aktuellen Aspekte der antiviralen Therapie vor und nach Transplantation zusammen. Von ganz aktuellem Interesse ist hierbei auch der Einsatz der seit einem Jahr verfügbaren HCV-Protease-Inhibitoren bei diesen Patienten.

Eine häufige Komplikation von Patienten mit Leberzirrhose ist die Entwicklung eines Leberzellkarzinoms (hepatocellular carcinoma, HCC) - weltweit einer der häufigsten menschlichen Tumoren mit steigender Inzidenz und Prävalenz. Die Therapie erfolgt stadienabhängig und beinhaltet lokal interventionelle, chirurgische und systemische Therapiemöglichkeiten. Bei der lokal interventionellen Therapie wurde in den letzten Jahren die Radioembolisation eingeführt. Ertle, Gerken und Schlaak [3], Essen, stellen die Mög-

\section{KARGER \\ Fax +497614520714 \\ Information@Karger.de}

www.karger.com
(C) 2012 S. Karger GmbH, Freiburg

$1662-6664 / 12 / 0285-0295 \$ 38.00 / 0$

Accessible online at:

www.karger.com/vim 
lichkeiten dieser Methoden in ihrem Beitrag ausführlich dar. Aus onkologischer Sicht stellt die Lebertransplantation für Patienten mit einem HCC die optimale Therapie dar, weil hierdurch sowohl die Tumorerkrankung saniert als auch der zugrunde liegende Risikofaktor «Leberzirrhose» beseitigt wird. Zangos, Ulrich, Eichler und Vogl [4], Frankfurt/M., stellen in ihrer Übersicht die Chancen und Risiken von sogenannten «Bridging»-Verfahren dar. Hierbei handelt es sich vor allem um die transarterielle Chemoembolisation, die eingesetzt wird, um zu verhindern, dass Tumoren während der Wartezeit auf einer Transplantationsliste weiter wachsen und so eine Lebertransplantation innerhalb der Mailand-Kriterien nicht mehr erlauben. Der Beitrag von Schnitzbauer, Woeste, Ulrich und Bechstein [5], Frankfurt/M., beleuchtet die immensen Fortschritte und die Standardisierung der Leber- und Multiorgantransplantationschirurgie in den letzten Jahren, die zu einer deutlich geringeren Morbidität und Mortalität geführt haben. Hierzu hat auch die perioperative Betreuung durch die Anästhesie, aber auch die intensivmedizinische Betreuung im Allgemeinen von Patienten mit fortgeschrittener Lebererkrankung beigetragen. Diese Aspekte werden von Bojunga und Friedrich-Rust [6], Frankfurt/M., ausführlich dargestellt und diskutiert.
Leider entwickeln etwa $20 \%$ der Patienten, die aufgrund eines HCC lebertransplantiert werden, ein Tumorrezidiv. Trojan und Welker [7], Frankfurt/M., fassen in ihrem Beitrag die aktuellen präventiven und therapeutischen Strategien für diese Situation zusammen.

Als Abschluss dieses Themenschwerpunkts der VISZERALMEDIZIN finden Sie das Interdisziplinäre Gespräch [8], das von Jörg Trojan, Frankfurt/M., koordiniert wurde. Wir konnten hierfür mit Guido Gerken, Essen, Peter Schirmacher, Heidelberg, und Thomas Vogl, Frankfurt/M., Experten der Fachdisziplinen Hepatologie, Pathologie und Radiologie gewinnen, die mit uns aktuelle Fragen zum Themenkomplex «Leberzirrhose und $\mathrm{HCC} \gg$ diskutieren.

Unser Wunsch ist es, mit diesem Schwerpunktheft darzustellen, dass die Betreuung von Patienten mit fortgeschrittener Leberzirrhose so interdisziplinär wie bei nur wenigen anderen Erkrankungen ist. Gerade die Fortschritte auf diesem Gebiet der Medizin wurden nur durch die enge Verzahnung der verschiedenen Fachdisziplinen möglich. Wir möchten daher aufzeigen, dass nur durch gelebte und auch offen kommunizierte Interdisziplinarität Vertrauen in die für unsere Patienten so wichtige Therapie in Form der Lebertransplantation geschaffen werden kann.

\section{Literatur}

1 Gerbes AL, Gülberg V: Aszites, Pfortaderthrombose und hepatische Enzephalopathie bei Leberzirrhose: Aktuelle Therapieempfehlungen. Viszeralmedizin 2012;28:297-303.

2 Farnik H, Sarrazin C: Behandlung von Lebertransplantationspatienten mit chronischer Virushepatitis. Viszeralmedizin 2012;28:304-310.

3 Ertle J, Gerken G, Schlaak JF: Radioembolisation bei fortgeschrittenem hepatozellulärem Karzinom. Viszeralmedizin 2012;28:311-316.
4 Zangos S, Ulrich F, Eichler K, Vogl TJ: Hepatozelluläres Karzinom: Bridging-Verfahren vor der Lebertransplantation. Viszeralmedizin 2012;28:317-323.

5 Schnitzbauer AA, Woeste G, Ulrich F, Bechstein WO: Indikationen und Komplikationen bei Multiorgantransplantationen. Viszeralmedizin 2012;28: 324-329.

6 Bojunga J, Friedrich-Rust M: Intensivmedizinisches Management bei Lebererkrankungen im Endstadium (MELD > 30). Viszeralmedizin 2012;28:330-337.
7 Welker MW, Trojan J: Rezidiv des hepatozellulären Karzinoms nach orthotoper Lebertransplantation: Nachsorge und therapeutische Optionen. Viszeralmedizin 2012;28:338-343.

8 Trojan J (Gesprächsleiter): Interdisziplinäres Management bei fortgeschrittener Leberzirrhose. Viszeralmedizin 2012;28:344-347. 